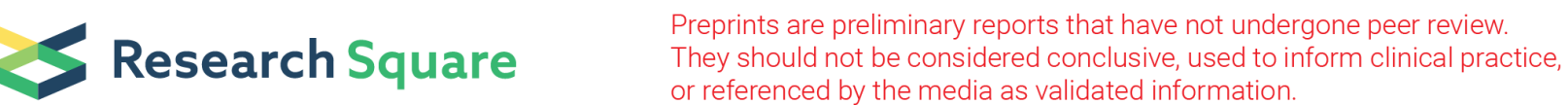

\section{Mutations in domain V of Mycoplasma pneumoniae 23S rRNA are not associated with clinical characteristics of M. pneumoniae pneumonia in children: a case control study}

\section{Huan Deng}

children hospital affiliated to Nanjing Medical Hospital https://orcid.org/0000-0003-3044-8546

Yifan Zhu

children's hospital affiliated to nanjing medical university

Jiamin Zhang

children's hospital affiliated to nanjing medical unversity

Qiangquan Rong

nanjing gaochun people's hospital

\section{Yao Quan}

nanjing gaochun people's hospital

Heng Tang

children's hospital Affiliated to nanjing medical university

Deyu Zhao ( $\square$ zhaodeyu988@126.com )

\section{Research}

Keywords: Mycoplasma pneumoniae, refractory Mycoplasma pneumoniae pneumonia, mutation, clinical characteristics

Posted Date: February 14th, 2020

DOI: https://doi.org/10.21203/rs.2.23539/v1

License: (c) (1) This work is licensed under a Creative Commons Attribution 4.0 International License. Read Full License 


\section{Abstract}

\section{Background}

Mycoplasma pneumoniae (MP) is a common agent of community-acquired pneumonia in children and young adults that can lead to refractory or persistent Mycoplasma pneumoniae pneumonia (MPP). Macrolide-resistant MP harbors point mutations in domain V of $23 \mathrm{~S}$ ribosomal Ribonucleic Acid (rRNA) with substitutions detected at positions 2063, 2064, 2067 and 2617. This study's purpose is to investigate the prevalence and clinical characteristics of mutations in domain V of MP 23S rRNA.

\section{Methods}

We sequenced the 23S rRNA domain V of MP strains collected from children with MPP. Clinical and laboratory data were also obtained, including gender, age, duration of fever, duration of fever after the start of macrolide therapy, MP-Deoxyribonucleic Acid (DNA) load at enrollment, leukocyte count, neutrophil count, and lymphocyte count, immunomodulators treatment and pulmonary complications.

\section{Results}

Of 276 strains, 255 (92.39\%) harbored A to G transition at the position 2063 (A2063G), and 21 (7.61\%) were not mutated. There were no significant differences in gender, age, duration of fever, duration of fever after the start of macrolide therapy, MP-DNA load at enrollment, hospitalization days, lymphocyte count and pulmonary complications when patients were stratified based on the presence or absence of domain $\checkmark$ mutations. We also found that children with refractory MPP experienced higher MP-DNA load than the non-refractory MPP, but the prevalence of domain $V$ mutations was comparable.

\section{Conclusions}

We found that clinical MP strains harbored very high mutation rate in 23S rRNA domain V, especially A2063G mutation. However, these mutations were not associated with clinical symptoms, laboratory results, pulmonary complications and development of refractory pneumonia. Instead, MP-DNA load was significantly different between refractory and non-refractory MPP.

\section{Background}

The prevalence of infection with Mycoplasma pneumoniae (MP) is widely underestimated, as most patients infected with MP are seldom symptomatic and rarely seek medical attention. MP is considered one common agent of community-acquired pneumonia in children and young adults. MP epidemics tend to cycle every 3 to 7 years[1], and infection may lead to refractory or life-threatening pneumonia with pulmonary and extrapulmonary complications[2-4].

As MP has no cell wall, it is intrinsically resistant to beta-lactams, glycopeptides, and fosfomycin antibiotics that target the cell wall. Therefore, macrolides, tetracyclines, and fluoroquinolones are used 
instead. Macrolides are considered as first-line agents in children to avoid potential age-related side effects of other therapies (tetracyclines have possible adverse effects on enamel hypoplasia and bone, and fluoroquinolones may influence the growth of bone and articular cartilage) [5, 6]. However, cases of refractory Mycoplasma pneumoniae pneumonia (MPP) have also steadily increased in recent years [2, 3].

Indeed, because macrolides inhibit protein synthesis by binding domain II and/or domain V of $23 \mathrm{~S}$ ribosomal Ribonucleic Acid (rRNA) in the 50S MP ribosomal subunit, several studies have demonstrated that genetic mutations in the $23 \mathrm{~S}$ rRNA can result in macrolide resistance in MP[6-9]. In particular, point mutations at nucleotide positions A2063, A2064, A2067 and C2617 in domain V, especially A to G transition at position 2063 (A2063G) and A to G transition at position 2064 (A2064G), confer strong resistance[10-12], whereas mutations at positions A2067 and C2617 confer lower levels of resistance[2, 6,12 ]. Notably, since 2000, the prevalence of macrolide-resistant MPP has rapidly increased, especially in Asian countries [6]. Worryingly one study suggests that macrolide resistance was found in more than $90 \%$ of Chinese MP isolates, which all harbored gene mutations [13]. Our previous research found that the morbidity of macrolide-resistant MP is even up to $92.45 \%$ [14]. The excessive use and misuse of macrolides may contribute to these mutations [15]. Refractory pneumonia was also increased, which showing no clinical or radiological response to macrolides, and could be easier to become severe or fatal pneumonia.

A growing body of researches have characterized the molecular epidemiology of MP infection. However, to our knowledge, rarely large-scale clinical research has to be conducted to characterize clinical strains of MP, and to investigate the clinical significance of mutations. In this, we amplified and sequenced the 23S rRNA domain V of a large number of clinical MP strains, and compared with the sequence in MP standard strain (M129). The aim was to investigate genotypes clinical MP strains on a large scale, determine the prevalence of domain $\mathrm{V}$ mutations, and evaluate the association between domain $\mathrm{V}$ mutations and clinical characteristics of MPP, especially refractory MPP.

\section{Methods}

\section{Study Population}

We enrolled patients admitted for MPP into the Department of Respiratory, Children's Hospital Affiliated to Nanjing Medical University from March 1, 2014 to May 31, 2015. Diagnosis pneumonia was based on symptoms at admission, including fever, cough, productive sputum, chest pain, dyspnea, abnormal breathing sounds, and radiographic pulmonary abnormalities that were at least segmental. In addition, acute MP infection was confirmed by Polymerase Chain Reaction (PCR) and serology (detecting MP IgM by enzyme-linked immunosorbent assay in acute phase), as well as by the absence of other pathogens (bacteria, other viruses, chlamydia pneumoniae, legionella pneumophila and so on) and the ineffectiveness of cephalosporin. All patients had both positive results for the above-mentioned MP tests and been diagnosed with pneumonia. We excluded convalescent patients, as well as patients with immunosuppressive illness, asthma, chronic lung disease, or other systematic disease. Complete medical 
records were obtained. Fever, hospitalization days, blood tests, MP-DNA load and chest radiography were noted, along with pulmonary complications including pulmonary atelectasis, pleuritis, and hydrothorax.

\section{Sample Preparation}

Two nasopharyngeal aspirates and serum samples were collected at admission. A portion of nasopharyngeal aspirates was assayed for MP-DNA load using the MP-DNA PCR kit (Acon Biotechnology, Zhejiang, China), following the manufacturer's instructions. The other nasopharyngeal aspirates were vigorously mixed with 4 volumes of $0.9 \%$ normal saline, incubated overnight at $4{ }^{\circ} \mathrm{C}$, and centrifuged at $13,500 \mathrm{rpm}$ and $4^{\circ} \mathrm{C}$ for $20 \mathrm{~min}$. The supernatant was discarded, and the pellet was resuspended in $40 \mu \mathrm{l}$ lysis solution (1\% Triton-100, Amresco, USA), and shaken for $10 \mathrm{~min}$ in a water bath at $100{ }^{\circ} \mathrm{C}$. Finally, lysates were clarified by centrifugation at $14,500 \mathrm{rpm}$ and $4{ }^{\circ} \mathrm{C}$ for $6 \mathrm{~min}$, and supernatants were retained.

\section{Gene Amplification}

$23 S$ rRNA domain $\mathrm{V}$ was amplified by nested PCR (Table 1). Amplification products, with expected size about $690 \mathrm{bp}$, were resolved on $1 \%$ agarose, visualized by ribonucleic acid staining, and imaged using a gel-imaging and analysis system. Amplification products were then shipped at $4{ }^{\circ} \mathrm{C}$ within 2 days for sequencing at Yingweijie, Shanghai, China. Finally, DNA sequences were compared using BLAST (http://blast.ncbi.nlm.nih.gov/Blast.cgi) to strain M129 (ATCC 29342).

Table 1

Primers for PCR and Sequencing

\begin{tabular}{|llll|}
\hline Primer & Sequence (5' to $\left.\mathbf{3}^{\prime}\right)$ & Position & Amplicon size (bp) \\
\cline { 1 - 3 } Forward-1 & GCAGTGAAGAACGAGGGG & $1758-1775$ & 942 \\
\cline { 1 - 3 } Reverse-1 & CACACTTAGATGCTTTCAGAG & $2680-2700$ & \\
\cline { 1 - 3 } Forward-2 & CGGTCCTAAGGTAGCGAAAT & $1963-1982$ & 690 \\
\cline { 1 - 3 } Reverse-2 & AACACTCTTCAATCTTCCTACGG & $2631-2653$ & \\
\hline
\end{tabular}

\section{Clinical Data, Treatment, and Diagnosis of Refractory MPP}

Clinical data were collected from medical records, including gender, age, fever, hospitalization days, immunomodulators, radiological findings, laboratory data, and MP-DNA load. Macrolide therapy strategies were $10 \mathrm{mg}$ azithromycin/ $\mathrm{kg} /$ day for the first three days in seven days, or with $30 \mathrm{mg}$ erythromycin $/ \mathrm{kg} /$ day. According to clinical manifestations, some drugs were selective used, such as expectorant, cough medicine, inhaled corticosteroid, bronchodilator and immunomodulators ( systemic corticosteroids and/or intravenous gamma immunoglobulin ). Interventional therapeutic fiber bronchoscopy or thoracentesis could be used in treatment of atelectasis or hydrothorax. Refractory MPP was defined as prolonged high degree fever $\left(>38.5^{\circ} \mathrm{C}\right.$ or $\left.103.3^{\circ} \mathrm{F}\right)$, worsening cough and (or) increasing infiltrates on chest radiographs after administration of macrolide antibiotic for 7 days or more. 


\section{Statistical Analyses}

Data were analyzed in IBM SPSS statistics 20.0. For data with skewed distribution, median values and range are reported. Student's t-test was used to compare continuous variables, while $\chi^{2}$ test and Fisher's exact test were used for categorical variables. A P value $<0.05$ was considered statistically significant.

\section{Results}

\section{Demographics}

A total of 276 patients were enrolled, of whom 137 (49.64\%) were male and 139 (50.36\%) were female. The median age was 4.93 years.

\section{MP 23S rRNA genotypes}

Of 276 strains, 255 (92.39\%) harbored the A2063G mutation in domain V, while the remaining 21 (7.61\%) did not contain mutations. One A2063G MP isolate strain contained an additional G insertion between position 2586 and 2587. And another one had three additional mutations including G-to-C transition at position 2601, $T$ insertion between position 2589 and 2590, and $T$ insertion between position 2612 and 2613 (Table 2).

Table 2

Mutations in MP 23S rRNA domain V

\begin{tabular}{|l|l|}
\hline Mutation & $\begin{array}{l}\text { Value }(n= \\
\text { 276) }\end{array}$ \\
\hline Only A2063G & $253(91.67 \%)$ \\
\hline A2063G and G insertion between 2586 and 2587 & $1(0.36 \%)$ \\
\hline $\begin{array}{l}\text { A2063G, G2601C, T insertion between 2589 and 2590, T insertion between } 2612 \text { and } \\
2613\end{array}$ & $1(0.36 \%)$ \\
\hline No mutation & $21(7.61 \%)$ \\
\hline
\end{tabular}

\section{Clinical Characteristics of infection with Mutated and Non-mutated MP strains}

Patients were stratified into two groups based on the presence or absence of mutations in the 23S rRNA domain $\mathrm{V}$ of MP. The mean age was 4.84 years in 255 patients (124 males, 131 females) infected with mutated MP, and 6.07 years in 21 patients (13 males, 8 females) infected with non-mutated MP strains. There were no significant differences in age and sex distribution, as well as in pulmonary complications, duration of fever, duration of fever after the start of macrolide therapy, MP-DNA load at enrollment, hospitalization days and immunomodulators treatment. However, leukocyte and neutrophil count were 
significantly higher in patients infected with mutated strains, although lymphocyte count was comparable between groups (Table 3).

\section{Table 3}

Clinical Characteristics of Infection with Mutated and Non-mutated MP Strains

\begin{tabular}{|c|c|c|c|}
\hline Variable & $\begin{array}{l}\text { Mutated } \\
(n=255)\end{array}$ & $\begin{array}{l}\text { Non-mutated } \\
(n=21)\end{array}$ & $P$ value \\
\hline Male/female & $124 / 131$ & $13 / 8$ & 0.242 \\
\hline Mean Age \pm SD (y) & $4.84 \pm 2.99$ & $6.07 \pm 3.49$ & 0.073 \\
\hline With/without pulmonary complications ${ }^{a}$ & $53 / 202$ & $4 / 17$ & 0.85 \\
\hline With/without immunomodulators & $56 / 199$ & $7 / 14$ & 0.233 \\
\hline \multicolumn{4}{|l|}{ Duration of fever (d) } \\
\hline Median (range) & $6(0-15)$ & $7(0-14)$ & \\
\hline Mean \pm SD & $6.23 \pm 3.76$ & $7.05 \pm 3.90$ & 0.342 \\
\hline \multicolumn{4}{|l|}{ Duration of fever after macrolide therapy (d) } \\
\hline Median (range) & $3(0-11)$ & $3(0-11)$ & \\
\hline Mean \pm SD & $3.65 \pm 2.83$ & $4.52 \pm 3.41$ & 0.184 \\
\hline \multicolumn{4}{|l|}{ MP-DNA load at enrollment ${ }^{b}$} \\
\hline Median (range) & $5.78(4.03-8.15)$ & $6.31(4.3-7.78)$ & \\
\hline Mean \pm SD & $5.96 \pm 1.08$ & $6.25 \pm 1.13$ & 0.233 \\
\hline \multicolumn{4}{|l|}{ Hospitalization days } \\
\hline Median (range) & $8(5-15)$ & $8(6-15)$ & \\
\hline Mean \pm SD & $8.78 \pm 2.48$ & $8.95 \pm 2.66$ & 0.233 \\
\hline Mean leukocyte \pm SD $(\times 109 / L)$ & $10.19 \pm 4.22$ & $8.14 \pm 2.56$ & 0.002 \\
\hline Mean neutrophil \pm SD $(\times 109 / L)$ & $5.73 \pm 3.55$ & $4.14 \pm 1.54$ & $<0.001$ \\
\hline Mean lymphocyte \pm SD $(\times 109 / L)$ & $3.46 \pm 2.23$ & $2.92 \pm 1.89$ & 0.278 \\
\hline
\end{tabular}

4.Clinical Characteristics of Refractory and Non-refractory MPP 
On the basis of the clinical and radiologic findings, 64 patients (29males, 35 females) were deemed to have refractory MPP, while 212 (108 males, 104 females) were considered to have non-refractory MPP. There was no significant difference in gender between groups. Notably, the mutation was found with comparable frequency between non-refractory (93.87\%) and refractory cases (87.5\%), $\mathrm{P}=0.092$.

Moreover, MP-DNA load at enrollment and age were significantly higher in patients with refractory MPP (Table 4).

Table 4

Clinical Characteristics of Non-refractory and Refractory MPP

\begin{tabular}{|c|c|c|c|}
\hline Variable & $\begin{array}{l}\text { Non-refractory } \\
(n=212)\end{array}$ & $\begin{array}{l}\text { Refractory } \\
(n=64)\end{array}$ & $P$ value \\
\hline Male/female & $108 / 104$ & $29 / 35$ & 0.43 \\
\hline Mean Age \pm SD (yr) & $4.36 \pm 2.89$ & $6.82 \pm 2.81$ & $<0.001$ \\
\hline With/without mutations & 199/13 & $56 / 8$ & 0.092 \\
\hline With/without pulmonary complications ${ }^{a}$ & $13 / 199$ & $44 / 20$ & $<0.001$ \\
\hline With/without immunomodulators & $15 / 197$ & $48 / 16$ & $<0.001$ \\
\hline \multicolumn{4}{|l|}{ Duration of fever (d) } \\
\hline Median (range) & $5(0-14)$ & $10(3-15)$ & \\
\hline Mean \pm SD & $5.00 \pm 3.05$ & $10.58 \pm 2.61$ & $<0.001$ \\
\hline \multicolumn{4}{|l|}{ Duration of fever after macrolide therapy (d) } \\
\hline Median (range) & $2(0-10)$ & $7.5(3-11)$ & \\
\hline Mean \pm SD & $2.61 \pm 2.05$ & $7.39 \pm 2.06$ & $<0.001$ \\
\hline \multicolumn{4}{|l|}{ MP-DNA load at enrollment ${ }^{b}$} \\
\hline Median (range) & $5.64(4.03-8.15)$ & $6.43(4.61-7.96)$ & \\
\hline Mean \pm SD & $5.83 \pm 1.10$ & $6.47 \pm 0.87$ & $<0.001$ \\
\hline \multicolumn{4}{|l|}{ Hospitalization days } \\
\hline Median (range) & $7(5-15)$ & $12(7-15)$ & \\
\hline Mean \pm SD & $7.86 \pm 1.74$ & $11.88 \pm 2.06$ & $<0.001$ \\
\hline
\end{tabular}

\section{Discussion}


Although MP infection shows typically self-limited course even without antibiotics, macrolides are still recommended as the first-line therapeutics for treating MP infection. However, a steadily increasing number of recent cases progress to refractory, severe, life-threatening MP pneumonia [1, 2]. Extensive use and misuse of macrolides may cause the rapid emergence of macrolide resistance [15]. Macrolides inhibit protein synthesis by binding to specific nucleotides of the 23S RNA in the 50S MP ribosomal subunit. Mutations at domain $\mathrm{V}$ of $23 \mathrm{~S}$ RNA reduce the affinity of the macrolides for the ribosome, which develop macrolide resistance [6-9]. Since 2000, some studies have confirmed that this microbiological problem is increasing throughout the world, although the highest prevalence has been observed in East Asia. The published rate was reported to be $87.2 \%$ in Korea, $81.6 \%$ in Japan, and up to $90 \%$ in China [6, 13]. Consistent with previous studies, we found that $92.39 \%$ clinical strains from children with MPP harbored A2063G mutation. Notably, we didn't detect mutations in the other spots at positions 2064, 2067, or 2617. However, several novel mutations were found, including a $\mathrm{G}$ to $\mathrm{C}$ transition at position 2601, a T insertion between positions $2589-2590$ and 2612-2613, and a $\mathrm{G}$ insertion between positions 2586-2587. Further studies are required to test whether these new mutations contribute to macrolide resistance.

Previous studies focus on the minimum inhibitory concentrations of macrolides in vitro, and explore point mutations of MP clinical strains [10-12]. However, the clinical relevance of these mutations had not been clearly characterized and large sample studies were fewer. In this study, patients with MPP infected by mutant strains in the clinical, laboratory and radiologic characteristics were similar with patients without mutations. These results hint the clinical symptoms, laboratory and radiologic characteristics of MPP are generally similar between mutations and without mutations. Previous studies on the comparisons of clinical manifestations between the groups also reported no significant differences $[14,16,17]$ As we all know, refractory MPP is characterized by long duration of fever, severe pulmonary inflammatory response. In this study, the prevalence of mutations in non-refractory and refractory MPP were $93.87 \%$ and $87.5 \%$, which suggested that the infection of mutant strains does not increase the refractoriness of MPP in children. In addition, some children infected mutant strain also were cured only treated by macrolide treatment. These reasons may be the anti-inflammatory of macrolides works synergistically, and use of immunomodulators improve prognosis regardless of macrolide resistance $[18,19]$. As previous research determined that the pathogenesis of MP are consist of direct damage mechanisms, immune damage and inflammatory damage[20] Thus, while there was some value in investigating the clinical significance of genetic mutations in MP, it is probably necessary to consider other risk factors that may trigger refractory, severe, or life-threatening pneumonia, such as a more robust host immune response with inflammatory cytokines, interleukins(IL), alexin, $\mathrm{CD}^{4+} \mathrm{T}$ cell and so on [21-23]. In refractory MPP, immunomodulators such as systemic corticosteroids or intravenous gamma immunoglobulin are considered to be an effective treatment option by reducing host inflammatory response $[18,19]$. Based on previous research, MP infection enhances mucin production and neutrophil recruitment, excretes inflammatory factors [20]. Mucus cell hypersecretion, especially goblet cell hyperplasia, has been shown in airways of MP infected mice [24]. Additionally, in children infected with MP, the expression levels of tumor necrosis factor-a (TNF-a), IL-1 $\beta$, IL-6, IL-10, C1q, C3,C4 in serum increase to varying degrees [21, 22]. 
Those cytokines, ILs and alexin maybe participate in some classical or bypass activation pathways, mediate inflammatory reaction and immune responses, and have various biological activities.

More interestingly, our study and Wang et al found that MP-DNA load at enrollment was significantly different between refractory pneumonia and non-refractory pneumonia groups [18], which maybe a risk factor of refractory MPP because of direct damage caused by increased MP load. The direct damage mechanisms of MP infection include adhesion damage, destruction of membrane fusion, invasive damage, and toxic damage [20]. This suggests that the higher and more persistent MP stimulation may induce a much stronger direct damage. To sum up, the occurrence of refractory MPP in children maybe largely depends on the interaction between MP and host immune response, regardless of mutations.

Our study is significant because it has compared the manifestations of MPP in children in a high macrolide resistance period for MP. But it also had several limitations. Firstly, our hospital is a tertiary hospital, and the enrolled patients had a much longer period prior to hospitalization at our hospital than in previous studies. Even this population may therefore have included some very severe MPP cases. Secondarily, our sample size was relatively small, and without multicenter research. Lastly, our study is that the MIC values were not measured.

\section{Conclusions}

This study provides the infecting MP strain in $92.39 \%$ of our patients harbor genetic mutations in the $23 \mathrm{~S}$ rRNA domain V. The A2063G mutation has been found in all mutated strains, which those new mutations need further study. Moreover, all results indicate that MPP infected by these mutations in 23S rRNA domain $\mathrm{V}$ were not specificity in clinical features, laboratory results, pulmonary complications, development of refractory pneumonia. Instead, we found that MP DNA load and host immune response may drive the development of refractory pneumonia.

\section{Abbreviations}

MP: Mycoplasma pneumoniae; MPP: Mycoplasma pneumoniae pneumonia; rRNA: ribosomal Ribonucleic Acid; DNA: Deoxyribonucleic Acid; A2063G: A to G transition at position 2063; A2064G: A to G transition at position 2064; PCR: Polymerase Chain Reaction; TNF-a: tumor necrosis factor-a; IL: interleukin;

\section{Declarations}

Ethics approval and consent to participate: The study protocol was approved by the ethics committee of Children's Hospital Affiliated to Nanjing Medical University. Participant consent was written by the legal representatives of patients and informed consent forms were achieved.

Consent for publication: The study has obtained consent to publish from children's parent or legal guardian. 
Availablility of data and materials: All data generated or analyzed during this study are included in this published article and available form the corresponding author on reasonable request.

Competing interests: The authors declare that they have no conflict of interest.

Funding: This study is supported by a grant from .

Authors' contributions: Huan Deng and Yifan Zhu participated in the design of the study and the statistical analysis. Huan Deng wrote and submitted the manuscript. Jiamin Zhang and Yao Quan participated in the sample preparation, gene magnification and sequence alignment. Qiangquan Rong participated in the design of the study and performed the statistical analysis. Heng Tang conceived of the study, participated in its design and helped to draft the manuscript. Deyu Zhao participated in the design of the study. All authors read and approved the final manuscript.

Acknowledgements: Not applicable.

Author details: ${ }^{1}$ Department of Respiratory, Children's Hospital Affiliated to Nanjing Medical University, No.72 Guangzhou Road, Nanjing, Jiangsu, China;

2 Department of Pediatrics, Nanjing Gaochun People's Hospital, No.9 Chunzhong Road, Chunxi Town, Gaochun District, Nanjing, Jiangsu, China.

\section{References}

1. Lee KY . Pediatric respiratory infections by Mycoplasma pneumoniae. Expert Rev Anti Infect Ther 2008; $6: 509-521$.

2. Zhou Y, Zhang Y, Sheng Y, Zhang L, Shen Z, Chen Z. More complications occur in macrolide-resistant than in macrolide-sensitive Mycoplasma pneumoniae Antimicrob Agents Chemother 2014;58:10341038 .

3. Azumagawa K, Kambara Y, Murata T, Tamai H. Four cases of arthritis associated with Mycoplasma pneumoniae Pediatr Int 2008;50:511-513.

4. Hawkins S, Rausch CM, McCanta AC. Constrictive pericarditis secondary to infection with Mycoplasma pneumoniae. Curr Opin Pediatr 2011;23:126-129.

5. Waites KB, Talkington DF. Mycoplasma pneumoniae and its role as a human pathogen. Clin Microbiol Rev 2004;17:697-728.

6. Waites KB, Xiao L, Liu Y, Balish MF, Atkinson TP. Mycoplasma pneumoniae from the Respiratory Tract and Beyond. Clin Microbiol Rev 2017;30:747-809.

7. Douthwaite S, Hansen LH, Mauvais P. Macrolide-ketolide inhibition of MLS-resistant ribosomes is improved by alternative drug interaction with domain II of 23S rRNA. Mol Microbiol 2000; 36 :183193 
8. Lucier TS, Heitzman K, Liu SK, Hu PC. Transition mutations in the 23S rRNA of erythromycin-resistant isolates of Mycoplasma pneumoniae. Antimicrob Agents Chemother 1995; 39 :2770-2773

9. Bébéar CM, Pereyre S. Mechanisms of drug resistance in Mycoplasma pneumoniae. Curr Drug Targets Infect Disord 2005; 5 :263-271

10. Morozumi M, Takahashi T, Ubukata K. Macrolide-resistant Mycoplasma pneumoniae. characteristics of isolates and clinical aspects of community-acquired pneumonia. J Infect Chemother 2010; 16 :7886.

11. Okazaki N, Narita M, Yamada S, Izumikawa K, Umetsu M, Kenri T, Sasaki Y, Arakawa Y, Sasaki T. Characteristics of macrolide-resistant Mycoplasma pneumoniae strains isolated from patients and induced with erythromycin in vitro. Microbiol Immunol 2001; 45:617-620

12. Morozumi M, Hasegawa K, Kobayashi R, Inoue N, Iwata S, Kuroki H, Kawamura N, Nakayama E, Tajima T, Shimizu K, Ubukata K. Emergence of macrolide-resistant Mycoplasma pneumoniae with a $23 S$ rRNA gene mutation. Antimicrob Agents Chemother 2005; 49 :2302-2306.

13. Xin D, Mi Z, Han X, Qin L, Li J, Wei T, Chen X, Ma S, Hou A, Li G, Shi D. Molecular mechanisms of macrolide resistance in clinical isolates of Mycoplasma pneumoniae from China. Antimicrob Agents Chemother 2009; 53 :2158-2159.

14. Deng H, Rui J, Zhao D, Liu F. Mycoplasma pneumoniae23S rRNA A2063G mutation does not influence chest radiography features in children with pneumonia. Int Med Res 2018; 46:150-157.

15. Cao B, Qu J-X, Yin Y-D and Van Eldere J. Overview of antimicrobial options for Mycoplasma pneumoniae pneumonia: focus on macrolide resistance. Clin Respir J 2015;00:000-000. DOI:10.1111/crj.12379.

16. Wang M, Wang Y, Yan Y, Zhu C, Huang L, Shao X, Xu J, Zhu H, Sun X, Ji W, Chen Z. Clinical and laboratory profiles of refractory Mycoplasma pneumoniae pneumonia in children. Int J Infect Dis 2014; 29:18-23.

17. Chen Y, Min F,Ling Y, Li G, Ye H, Pan J, Wang L, Xie Y. Clinical Characteristics and Antibiotic Resistance of Mycoplasma Pneumoniae Pneumonia in Hospitalized Chinese Children. Comb Chem High Throughput Screen 2018; 21:749-754.

18. Miyashita N, Kawai Y, Inamura N, Tanaka T, Akaike H, Teranishi H, Wakabayashi T, Nakano T, Ouchi K, Okimoto N. Setting a standard for the initiation of steroid therapy in refractory or severe Mycoplasma pneumoniae pneumonia in adolescents and adults. J Infect Chemother 2015 ; 21 :153160.

19. Luo Z, Luo J, Liu E, Xu X, Liu Y, Zeng F, Li S, Fu Z. Effects of prednisolone on refractory Mycoplasma pneumoniae pneumonia in children. Pediatr Pulmonol 2014; 49 :377-380.

20. He J, Liu M, Ye Z, Tan TP, Liu XH, You XX, Zeng YH, Wu YM. Insights into the pathogenesis of the mycoplasma pneumoniae(review).MOL MED REP 2016;14:4030-4036

21. Pang HX, Qiao HM, Cheng HJ, Zhang YF, Liu XJ and Li JZ. Levels of TNF-alpha, IL-6 and IL-10 in bronchoalveolar lavage fluid in children with Mycoplasma pneumoniae pneumonia. Zhongguo Dang Dai Er Ke Za Zhi 13: 808-810, 2011 (In Chinese) 
22. Loos $\mathrm{M}$ and Brunner $\mathrm{H}$. Complement components $(\mathrm{C} 1, \mathrm{C} 2, \mathrm{C} 3, \mathrm{C} 4)$ in bronchial secretions after intranasal infection of guinea pigs with Mycoplasma pneumoniae: Dissociation of unspecifc and specifc defense mechanisms. Infect Immun 25: 583-585, 1979.

23. Ye Q, Xu XJ, Shao WX, Pan YX and Chen XJ. Mycoplasma pneumoniae infection in children is a risk factor for developing allergic diseases. Scientific World Journal 2014: 986527, 2014.

24. Kraft, M., et al., Mycoplasma pneumoniae induces airway epithelial cell expression of MUC5AC in asthma. Eur Respir J, 2008. 31(1): p. 43-6 